phocyte infusions (15). Retaining the GVL effect while eliminating GVHD has been an elusive clinical goal. Additional testing is needed to determine whether CD $44^{+} \mathrm{CD} 62 \mathrm{~L}^{-} \mathrm{CD} 4^{+}$effector memory $\mathrm{T}$ cells might retain GVL activity, and whether specific immunization strategies of the donor might enhance transfer of protective immunity and GVL.

\section{Translation to clinical testing}

Finally, as pointed out by the authors (5), "if these murine results are applicable to human alloSCT, selective administration of memory $T$ cells could greatly improve post-transplant immune reconstitution." Before this is attempted clinically, murine $\mathrm{CD} 44^{+} \mathrm{CD} 62 \mathrm{~L}^{-}-$ $\mathrm{CD}^{+}$effector memory $\mathrm{T}$ cells still need to be tested in other strain combinations, evaluated for transfer of protective immunity, and tested for GVL potential. In addition, the immune capabilities of human $\mathrm{CD} 44^{+} \mathrm{CD} 62 \mathrm{~L}^{-}-$ $\mathrm{CD}^{+}$effector memory $\mathrm{T}$ cells must be studied in vitro and in adoptive transfer models (i.e., in immunodeficient or humanized mice) to determine whether their behavior parallels that of murine CD $44^{+} \mathrm{CD} 62 \mathrm{~L}^{-} \mathrm{CD} 4^{+} \mathrm{T}$ cells.

There is still much to do before we can forget about GVHD. The work of Anderson et al. (5) may be an important step toward that goal.

\section{Acknowledgments}

I.N. Buhtoiarov is supported by an International Union against Cancer-American Cancer Society international fellowship for beginning investigators.

1. Bach, F.H., Albertini, R.J., Joo, P., Anderson, J.L., and Bortin, M.M. 1968. Bone-marrow transplantation in a patient with the Wiskott-Aldrich syndrome. Lancet. 2:1364-1366.

2. Hong, R., et al. 1968. Immunological restitution in lymphopenic immunological deficiency syndrome. Lancet. 1:503-506.

3. Trigg, M.E., et al. 1985. Clinical trial depleting $T$ lymphocytes from donor marrow for matched and mismatched allogeneic bone marrow transplants. Cancer. Treat. Rep. 69:377-386.

4. Mackall, C., and Gress, R. 1997. Pathways of T-cell regeneration in mice and humans: implications for bone marrow transplantation and immunotherapy. Immunol. Rev. 157:61-72.

5. Anderson, B.E., et al. 2003. Memory CD4 ${ }^{+} \mathrm{T}$ cells do not induce graft-versus-host disease. J. Clin. Invest. 112:101-108. doi:10.1172/JCI200317601.
6. Leng, Q., and Bentwich, Z. 2002. Beyond self and nonself: fuzzy recognition of the immune system. Scand. J. Immunol. 56:224-232.

7. Mandell, R.B., and Sondel, P.M. 1985. Allele-specific alien-driven diversity, immune responsiveness and MHC-restriction. Immunol. Today. 6:321-323.

8. Lanzavecchia, A., and Sallusto, F. 2000. Dynamics of $\mathrm{T}$ cell lymphocyte responses: intermediates, effectors and memory cells. Science. 290:92-97.

9. Sallusto, F., Lenig, D., Reihold, F., Lipp, M., and Lanzavecchia, A. 1999. Two subsets of memory T cells with distinct homing potentials and effector functions. Nature. 401:708-712.

10. Hengel, R.L., et al. 2003. Cutting edge: L-selectine (CD62L) expression distinguishes small resting memory CD4+ T cells that preferentially respond to recall antigen. J. Immunol. 170:28-32.

11. Hogan, R.J., et al. 2001. Protection from respiratory virus infections can be mediated by antigen-specific CD4(+) $\mathrm{T}$ cells that persist in the lung. J. Immunol. 193:981-986.

12. Andersen, P., and Smedegaard, B. 2000. CD4+ T-cell subsets that mediate immunological memory to Mycobacterium tuberculosis infection in mice. Infect. Immun. 68:621-629.

13. Horowitz, M.M., et al. 1990. Graft-versus-leukemia reactions after bone marrow transplantation. Blood. 75:555-562.

14. Sondel, P.M. 2000. The graft vs. leukemia effect. In Allogeneic immunotherapy for malignant diseases. J. Barrett and Y.Z. Jiang, editors. Marcel Dekker Inc. New York, New York, USA. 1-12.

15. Slavin, S., et al. 1996. Allogeneic cell therapy with donor peripheral blood cells and recombinant human interleukin-2 to treat leukemia relapse after allogeneic bone marrow transplantation. Blood. 87:2195-2204.

\title{
Preventing pathological regression of blood vessels
}

\section{Eli Keshet}

Department of Molecular Biology, The Hebrew University-Hadassah Medical School, Jerusalem, Israel

\begin{abstract}
Oxygen administration to premature infants suppresses retinal VEGF expression and results in the catastrophic vessel loss associated with retinopathy of prematurity. A study investigating the development of the retinal vasculature in mice (see related article on pages 50-57) demonstrates that specific activation of VEGF receptor-1 by placental growth factor-1 protects against oxygen-induced vessel loss without stimulating vascular proliferation and neovascularization.
\end{abstract}

J. Clin. Invest. 112:27-29 (2003). doi:10.1172/JCI200319093.

\footnotetext{
Address correspondence to: Eli Keshet, Department of Molecular Biology,

The Hebrew University-Hadassah Medical School, Jerusalem 91120, Israel.

Phone: 972-2-6758496; Fax: 972-2-6757195; E-mail: keshet@cc.huji.ac.il.

Conflict of interest: The author has declared that no conflict of interest exists.

Nonstandard abbreviations used: retinopathy of prematurity (ROP); VEGF receptor-1 (VEGFR-1, also known as Flt-1); VEGF receptor-2 (VEGFR-2, also known as Flk-1); placental growth factor (PlGF); extracellular signal-regulated kinase (ERK).
}

A number of human disorders are associated with obliteration of preexisting blood vessels. Microvessel rarefaction often takes place in the hypertensive lung, in the myocardium of patients with chronic renal failure, and in the elderly. Conversely, a failure to eliminate transient embryonic vasculature destined for regression may lead to a disease, as exemplified by the common congenital developmental anom- aly of the eye, persistent hyperplastic primary vitreous, in which hyaloid vessels fail to regress. A striking example of a disease caused by vessel regression is retinopathy of prematurity (ROP). ROP is a blindness-causing neovascularizing disease that affects premature infants treated with high concentrations of oxygen. ROP develops in two distinct stages. First, the hyperoxic insult leads to obliteration of immature retinal vessels, thereby compromising retina perfusion. The second phase, initiated upon resumption of the breathing of normal air, is an adverse compensatory neovascularization response, mediated by ischemiainduced VEGF, in which formation of new vessels is excessive, neovessels are leaky, and the inner limiting membrane of the retina is breached, allowing vessel growth into the vitreous. The later event may ultimately lead to retinal detachment and vision loss.

\section{Protecting retinal vessels from oxygen-induced obliteration}

Why are newly formed blood vessels of the retina so vulnerable to excess oxygen? Vessel regression in ROP repre- 
sents an exaggeration of an otherwise natural response to oxygen surplus. Normally, the tissue responds to excess oxygen by trimming its microvasculature to the extent that oxygen supply is reset to match the metabolic requirements of the tissue. However, the vast excess of iatrogenic oxygen is misinterpreted and results in overpruning of the newly formed vascular tree. The process is mediated by VEGF acting as a vascular survival factor. Specifically, suppression of VEGF expression by hyperoxia to levels below those required for maintaining immature vessels leads to endothelial cell apoptosis (1). It was further demonstrated that vessels can indeed be rescued by exogenous VEGF compensating for the diminution of endogenous VEGF, suggesting that VEGF might be used therapeutically for preventing vessel regression in ROP (1). This proposition seemed counterintuitive, however, as VEGF is also the factor that stimulates abnormal vessel proliferation in ROP and, furthermore, VEGF might induce vascular leaks. In this issue of the JCI, Shih and coworkers have circumvented these concerns by using an agonist that exclusively activates the VEGF receptor-1 (VEGFR-1, also known as Flt-1) namely, the placental growth factor-1 (PlGF-1) (2). The authors show that PlGF-1 protects neonatal retina vessels from hyperoxia-induced obliteration without provoking retinal neovascularization or edema (2) (Figure 1).

\section{Differential effects of VEGF and PIGF}

Unlike VEGF, which interacts with both VEGFR-1 and VEGFR-2 (also known as Flk-1), PlGF only binds VEGFR-1 (3). PIGF and VEGFR-1 were, until recently, neglected as potential therapeutic targets, since PlGF and the kinase activity of VEGF-R1 were found to be dispensable for blood vessel formation in the embryo (4). This situation changed with recent findings that PlGF may stimulate angiogenesis in the adult with at least a comparable efficiency to that of VEGF and, conversely, that specific inhibition of VEGFR-1 suppress neovascularization in tumors and the ischemic retina $(5,6)$. The question of why a PlGF/VEGFR-1 interaction may have a profound effect on pathophysiological angiogenesis but not on developmental neovascularization remains enigmatic. Several mutually nonexclusive mechanisms for the differential effects of PIGF and VEGFR-1 have been proposed. For example, the relative abundance of VEGF and PlGF may determine the pattern of receptor occupancy, and, similarly, the relative abundance of VEGFR-1 and VEGFR-2 may determine the nature of signals transmitted. Additionally, the relative amounts of these proteins determine the probability of making inactive PlGF/VEGF heterodimers (7) or VEGFR-1/VEGFR-2 heterodimers with altered ligand-binding properties. Other levels of complexity reside in the expression of VEGFR-1 and VEGFR-2 by nonendothelial cells, a possible cross-talk between VEGFR-2 and VEGFR-1 homodimers, differential modulation of PlGF-1 and PlGF-2 by accessory neuropilin receptors, and sequestration of both PlGF and VEGF by soluble VEGFR-1 receptors (8). Thus, the integrated net effect of PlGF is likely to be contextual, depending on the exact stoichiometries of all these players. In this regard, the particular constellation present in the hyperoxic neonatal retina, i.e., suppressed production of VEGF and predominance of VEGFR-1 expression by endothelial cells (2), appears conducive to a PlGFinduced pro-survival signal but not to PlGF-induced proliferation.

The study by Shih et al. (2) highlights an emerging role of PlGF-1 as a vascular survival factor. A vasoprotective effect of PlGF against oxygeninduced degeneration of retinal vessels was also reported by Upalakalin et al. (9). Previously, the vasoprotective effect of PlGF was demonstrated in tumors (10), and its activity as an autocrine survival factor for trophoblasts was also demonstrated (11). Mechanistically, little is known regarding the signal transduction pathways that culminate in vasoprotection. In the case of the pro-survival signals elicited by VEGF, a phosphorylation of the survival kinase Akt1 and the functional significance of this reaction in inhibiting endothelial cell apoptosis have been demonstrated $(12,13)$. Additionally, VEGF was shown to

\footnotetext{
Figure 1

ROP pathogenesis and suggested treatments. (a) Retina vessels in the process of their formation and progressive covering of the retina surface. (b) Hyperoxia at this formative stage suppresses VEGF and, consequently, results in regression of newly formed vessels. (c) Upon return to normal air, the ischemic retina upregulates VEGF to high levels, causing excessive formation of leaky vessels. To antagonize VEGF at this stage has been suggested as a strategy to reduce adverse vessel formation. (d) An alternative strategy proposed by Shih et al. (2) is to protect retina vessels from oxygen-induced obliteration through administration of PIGF-1.
}

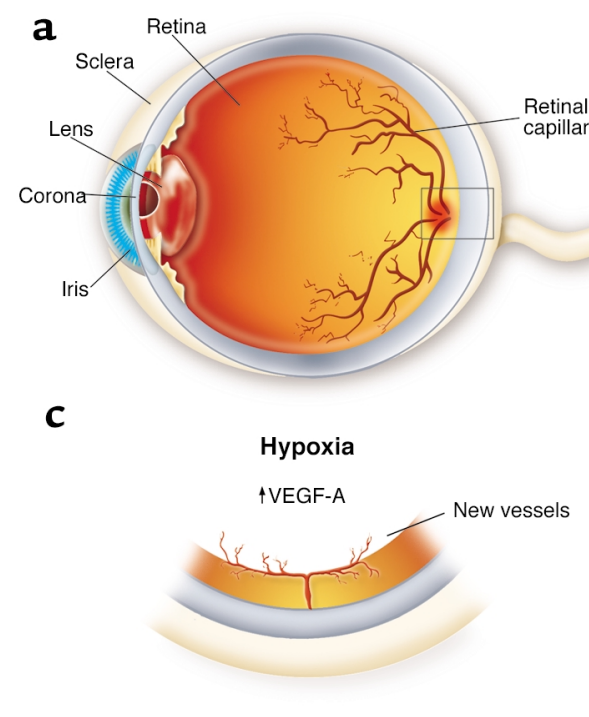

b
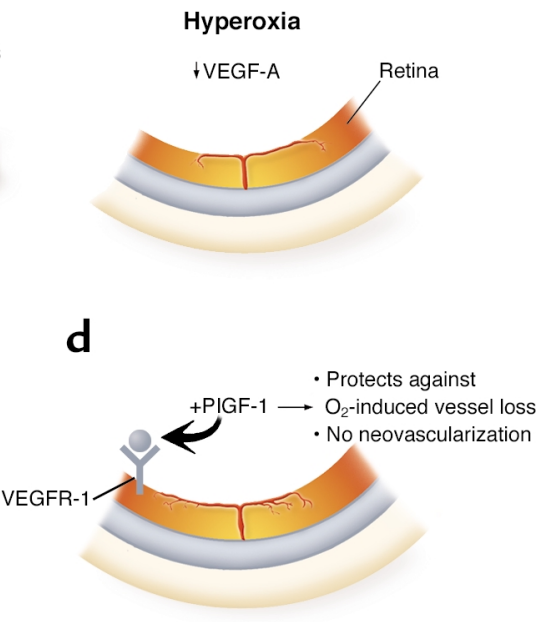
upregulate expression of antiapoptotic genes like the caspase inhibitor survivin, and Bcl-2 $(14,15)$. For PlGF, the stress-activated protein kinase, JNK, and P38 kinase pathways were shown to be activated in trophoblasts, while the extracellular signal-regulated kinase-1 (ERK-1) and ERK-2 pathways were activated in endothelial cells (11). Also, PlGF was shown to specifically upregulate survivin (10).

\section{Prospects of vessel-stabilization therapy}

What are the prospects of harnessing vessel-stabilizing agents for therapy? Clearly, the case of ROP is unique, considering the defined nature of the pathogenic insult, its relatively short duration (unlike in the chronic cases of vessel rarefaction, vessels in ROP need to be protected only during the period spent in the oxygen chamber), and its predetermined onset. These characteristics, in conjunction with the facts that the vitreous is a close, immunoprivileged compartment and that the superficial retinal vessels are accessible to injected reagents, increase the likelihood of success. An alternative approach to growth factor administration is the use of mimetics that activate the relevant signal transduc- tion pathways. This, however, must await further mechanistic insights on transduction of pro-survival signals. ROP is different from other pathologies of abnormal vessel regression in that the vessels eliminated are immature and hence VEGF dependent. Immaturity of vessels in expanding tumors may also be exploited for enforcing vessel regression via a VEGF withdrawal strategy (16). There are no clues, however, on how the rarefaction of mature vessels that are refractory to VEGF might be prevented.

1. Alon, T., et al. 1995. Vascular endothelial growth factor acts as a survival factor for newly formed retinal vessels: implications for retinopathy of prematurity. Nat. Med. 1:1024-1028.

2. Shih, S.-C., Ju, M.-H., Liu, N., and Smith, L.H.H 2003. Selective stimulation of VEGFR-1 prevents oxygen-induced retinal vascular degeneration in retinopathy of prematurity. J. Clin. Invest. 112:50-57. doi:10.1172/JCI200317808.

3. Persico, M.G., Vincenti, G., and DiPalma, T. 1999. Structure, expression and receptor-binding properties of placenta growth factor (PlGF). Curr. Top. Microbiol. Immunol. 237:31-40.

4. Hiratsuka, S., Minowa, O., Kuno, J., Noda, T., and Shibuya, M. 1998. Flt-1 lacking the tyrosine kinase domain is sufficient for normal development and angiogenesis in mice. Proc. Natl. Acad. Sci. U. S. A. 95:9349-9354.

5. Carmeliet, P., et al. 2001. Synergism between vascular endothelial growth factor and placenta growth factor contributes to angiogenesis and plasma extravasation in pathological conditions. Nat. Med. 7:575-583.

6. Luttun, A., et al. 2002. Revascularization of ischemic tissues by PLGF treatment, and inhibition of tumor angiogenesis, arthritis and atherosclerosis by anti-flt1. Nat. Med. 8:831-840.

7. Eriksson, A., et al. 2002. Placenta growth factor-1 antagonizes VEGF-induced angiogenesis and tumor growth by the formation of functionally inactive PlGF-1/VEGF heterodimers. Cancer Cell. 1:99-108.

8. Maynard, S.E., et al. 2003. Excess placental soluble fms-like tyrosine kinase 1 (sFlt1) may contribute to endothelial dysfunction, hyperten sion, and proteinuria in preeclampsia. J. Clin. Invest. 111:649-658. doi:10.1172/JCI200317189.

9. Upalakalin, J.N., Hemo, I., Dehio, C., Keshet, E., and Benjamin, L.E. 2002. Survival mechanisms during vascular remodeling. Cold Spring Harbor Symp. Quant. Biol. 67:181-187.

10. Adini, A., Kornaga, T., Firoozbakht, F., and Benjamin, L.E. 2002. Placental growth factor is a survival factor for tumor endothelial cells and macrophages. Cancer Res. 62:2749-2752.

11. Desai, J., Holt-Shore, V., Torry, R.J., Caudle, M.R., and Torry, D.S. 1999. Signal transduction and biological function of olacenta growth factor in primary human trophoblast. Biol. Reprod. 60:887-892.

12. Gratton, J.P., et al. 2001. Akt down-regulation of p38 signaling provides a novel mechanism for vascular endothelial growth factor-mediated cytoprotection in endothelial cells. J. Biol. Chem. 276:30359-30365.

13. Shiojima, I., and Walsh, K. 2002. Role of Akt signaling in vascular homeostasis and angiogenesis. Circ. Res. 90:1243-1250.

14. Gerber, H.P., Dixit, V., and Ferrara, N. 1998. Vascular endothelial growth factor induces expression of the antiapoptotic proteins $\mathrm{Bcl}-2$ and A1 in vascular endothelial cells. J. Biol. Chem. 273:13313-13316.

15. O'Connor, D.S., et al. 2000. Control of apoptosis during angiogenesis by survivin expression in endothelial cells. Am. J. Pathol. 156:393-398.

16. Benjamin, L.E., Golijanin, D., Itin, A., Pode, D., and Keshet, E. 1999. Selective ablation of immature blood vessels in established human tumors follows vascular endothelial growth factor withdrawal. J. Clin. Invest. 103:159-165. 